\title{
The review multidisciplinary meeting: un anno di letteratura sulla calcolosi renale Letteratura Dietologica
}

\section{E. Lenci}

Fondazione S. Maugeri, Clinica del Lavoro e della Riabilitazione, IRCCS, Istituto Scientifico di Veruno (NO)

La calcolosi renale si caratterizza per l'elevato numero di recidive.

Questo dato suggerisce il ruolo fondamentale svolto dai programmi di prevenzione medica, come è stato confermato da numerosi studi sull'impiego di trattamenti conservativi e farmacologici (1).

La ricerca nelle banche dati biomediche (2) suggerisce che, in base alla forma di litiasi, vi sono delle indicazioni dietetico-terapeutiche mirate al trattamento a seconda dei reperti urinari che il paziente presenta o del quadro clinico (ad esempio, se il pH sia acido o basico, del calcio urinario, o delle escrezioni di ossalato e citrato).

Non sempre le evidenze scientifiche possono essere utilizzate e facilmente implementate, tuttavia c'è un forte accordo in letteratura nel sostenere che questi cambiamenti debbano passare obbligatoriamente attraverso l'educazione terapeutica del paziente (3). Una ricerca sui termini o i modelli educazionali effettuata su Google fornisce ben 48.500 voci e 905 review su PubMed.

Il tema fondamentale del nuovo approccio terapeutico è il "chronic care model" (4).

L'approccio multidisciplinare è stato dimostrato da numerosi articoli e metanalisi e comprende un follow-up stretto, educazione del paziente e dei suoi familiari e visite domiciliari da parte di staff specialistico.

L'obiettivo per ottenere dei risultati accettabili con le risorse disponibili è quello di garantire:

- una cura efficace ed efficiente;

- un supporto all'autogestione;

- un regolare follow-up da parte del team;

- una cura che il paziente sia in grado di comprendere, condividere e che sia in accordo con il suo background culturale.

È del tutto ovvio quindi che un approccio multisciplinare non può esimersi dal coinvolgimento della figura del dietista con competenze specifiche. Purtroppo però, tale figura è latitante nel panorama assistenziale italiano.

La maggior parte dei Centri di Nefrologia e Dialisi come evidenziato dal Censimento dei Centri di Nefrologia e Dialisi promosso dalla SIN oltreché dal Progetto Nephrontieres, non ha dietisti dedicati a questa patologia, anche se l'educazione alimentare del paziente dovrebbe essere comunque garantita da modelli standard di trattamento che comprendano le regole universali per il trattamento della litiasi renale.

Dall'analisi comparativa della letteratura, emerge la necessità non solo di un'elaborazione di indicazioni standard, ma che esse diventino patrimonio comune da condividere con tutti i dietisti e per tutti i vari tipi di diete o norme dietetiche associate della calcolosi renale.

L'elaborazione di un piano dietoterapico comune dovrebbe comprendere:

- apporto di 2-3 litri di acqua al di;

- riduzione dell'apporto di sodio;

- riduzione dell'apporto di proteine animali.

\section{L'acqua}

Sin dai tempi di Ippocrate, è noto il vantaggio di mantenere un elevato volume urinario nei pazienti con litiasi reno-ureterale.

Studi recenti (5) confermano che la litiasi ricorrente di varia eziologia, può essere controllata con semplici aggiustamenti dietetici ed in particolare, con un'assunzione di liquidi sufficiente a mantenere una diuresi giornaliera di $2500 \mathrm{cc}$.

Tra le raccomandazioni per le patologie renali, citate nell'articolo, si prescrive un introito idrico giornaliero superiore ai 2 litri.

Facendo riferimento al Chronic Care Model, è importante fornire al paziente tutte le strategie idonee a garantire 
un'assunzione giornaliera di 2,5 litri di liquidi come ad esempio:

- bere al di fuori dei pasti;

- bere durante tutto il corso della giornata e

- prima del riposo notturno, in modo che i reni abbiano continuamente la necessaria quantità di acqua per sciogliere i sali da espellere;

- cuocere le verdure, che sono particolarmente ricche di ossalati in acqua con l'aggiunta di aceto. Quest'ultimo facilita l'eliminazione nell'acqua di cottura, di notevoli quantità di tali sostanze.

Questi accorgimenti permettono non solo di ridurre notevolmente i fattori di rischio della calcolosi, ma anche le recidive della malattia.

\section{Le proteine}

La dieta normale della popolazione italiana (e ancor peggio quella statunitense), è una dieta iperproteica.

Il consumo abituale di proteine si attesta intorno a circa 1.5 gr di proteine $/ \mathrm{kg} / \mathrm{die}$, ben lontani pertanto, dai livelli raccomandati dall'OMS di $1.0 \mathrm{gr}$ di proteine $/ \mathrm{kg}$. Inoltre un basso consumo di proteine soprattutto quelle di provenienza animale è considerato essere salutare (6). E quindi importante fornire ai pazienti affetti da calcolosi renale in cui il consumo proteico è un fattore di rischio (7), dopo aver condotto un'adeguata intervista dietetica sui consumi abituali di alimenti, alcuni dei consigli per ridurre l'apporto proteico a $1.0 \mathrm{gr}$ di proteine $/ \mathrm{kg} / \mathrm{die}$.

\section{Sodio}

Consigliare il paziente ad escludere il sale dalla tavola, non è mai un buon rimedio. Il sale rende sapidi i cibi ed è uno dei nutrienti che più facilmente è "nascosto" alla vista se non al palato. Per scoraggiare veramente l'utilizzo improprio del sale è necessario assistere il paziente indirizzando la sua scelta a sostitutivi più naturali quali erbe aromatiche, provenzali, spezie ecc.

Il paziente deve essere accompagnato ad un attento controllo nella lettura dell'etichetta nutrizionale dei prodotti commerciali, dove spesso non è del tutto segnalato in modo veritiero, il contenuto di sodio e di grassi (8), oltreché essere erudito sul contenuto di questo elettrolita negli alimenti (9).

Ma un punto fondamentale è che tutti i sanitari, dai medici agli infermieri ai dietisti non sono sempre del tutto consci ed attenti ai danni del sale e alle strategie atte a ridurlo.

In letteratura sono stati fatti molti tentativi come il "Blog del sale in ambito nefrologico" $(10,11)$, consigli e raccomandazioni per la popolazione normale (12-14) e per i cardiopatici (15) che sono una popolazione in grande aumento e a forte rischio di morbidità.

\section{Conclusioni}

L'analisi della letteratura porta quindi a due filoni ben distinti e di diversa importanza ed impatto sociale:

-il filone di scoperte e suggerimenti per piccoli gruppi di popolazione affetta da particolari tipi di litiasi e quindi con cambiamenti dietetici a carico spesso di singoli nutrienti;

- il filone di profilassi e prevenzione della popolazione normale che, seppur con piccoli ma significativi cambiamenti, come abbiamo visto dall'analisi presentata, può avere un grande impatto sulla riduzione del rischio litogeno in generale.

E necessario però, ed è questo il messaggio che ci sentiamo di lasciare, basato su dati della letteratura sempre più imponenti e pesanti, che i primi a dover sapere e trasmettere il messaggio debbano essere i sanitari. Da come e quanto sapranno essere convincenti, persuasivi e compartecipativi dipenderà non solo il successo della terapia, ma soprattutto il recepimento di alcuni principi nelle leggi e consuetudini dei vari stati (16-18).

\section{Indirizzo degli Autori:}

Elisabetta Lenci, MD

Dietista, Fondazione S. Maugeri

Clinica del Lavoro e della Riabilitazione

IRCCS, Istituto Scientifico

Veruno (Novara) Italy

elisabetta.lenci@fsm.it 


\section{Bibliografia}

1. Consensus Conference. Prevention and Treatment of Kidney Stones JAMA 1988; 260: 978-81.

2. Grases F, Antonia Costa-Bauza A, and Prieto MR. Renal lithiasis and nutrition. Nutrition Journal 2006; 5: 23.

3. Flagg LR. Dietary and holistic treatment of recurrent calcium oxalate kidney stones: review of literature to guide patient education. Nurs 2007; 27 (2): 113-22.

4. Wagner, et al. The Chronic Care Model "evidence- based model" by the MacColl Institute ACP-ASIM Journals and Books.

5. Ulrich O. Wenzel, Lee A, Hebert, Rolf AK Stahl, and Ingo Krenz. My doctor said I should drink a lot! Recommendations for fluid intake in patients with Chronic Kidney Diseses. Clin J Nephrol 2006; 1: 344-6.

6. Red and Processed Meat Intake Linked to Mortality. Arch Intern Med 2009; 169: 543-5, 562-71.

7. Borghi L, et al. Comparison of Two Diets for the Prevention of Recurrent Stones in Idiopathic Hypercalciuria NEJM Volume 346: 77-84 January 10, 2002.

8. Supermarket food ë contains more fat and salt than quoted on label The Indipendent On Sunday 13 January 2008.

9. Educazione alimentare: le iniziative delle associazioni e del Ministero "Per togliere la saliera dalla tavola bisogna cambiare il gusto". Corriere della Sera 10 febbraio 2008.

10. The salt Blog http://www.thesaltblog.org/sites/0/EN/ default.tpl
11. Shaldon S and Vienken J. The long forgotten salt factor and the benefits of using a 5-g-salt-restricted diet in all ESRD patients. Volume 23, Number 7, pp. 2118-20.

12. Consensus Action on Salt and Health (CASH): http:// www.actiononsalt.org.uk/

13. Selmer R, et al. Cost and health consequences of reducing the population intake of salt. J Epidemiol Community Health 2000; 54(9): 697-702.

14. Hooper, L., Bartlett, C., Davey Smith, G., Ebrahim, S. Systematic review of long term effects of advice to reduce dietary salt in adults. BMJ 2002; 325: 628.

15. Cappuccio FP. Salt and cardiovascular disease Reducing intake improves cardiovascular outcomes but few countries have effective policies. BMJ 2007; 334: 859-60.

16. World Action on Salt and Health (WASH) http://www. worldactiononsalt.com/index.htm

17. The UK's Food Standard's Agency (FSA) has set lower targets for the food industry to reach by 2012. http://www. food.gov.uk/news/pressreleases/2009/may/salttargets

18. Norwy: A gradual reduction in the average intake of salt to $5 \mathrm{~g}$ /day is desirable in the long term. In a shorter time perspective the population target is $6 \mathrm{~g} / \mathrm{d}$ for women and $7 \mathrm{~g} / \mathrm{d}$ for men. The salt intake should also be limited for children. For children below 2 years of age salt intake should not exceed $1.25 \mathrm{~g} / \mathrm{MJ}$ ( $239 \mathrm{kcal}$ ), in order to prevent children becoming accustomed to a diet with a high salt content. http://www.worldactiononsalt.com/action/ norway.doc 